\title{
Local Control of Extracellular Dopamine Levels in the Medial Nucleus Accumbens by a Glutamatergic Projection from the Infralimbic Cortex
}

\author{
César Quiroz, ${ }^{1}$ Marco Orrú, ${ }^{1}$ William Rea, ${ }^{1}$ Andrés Ciudad-Roberts, ${ }^{1}$ Gabriel Yepes, ${ }^{1}$ Jonathan P. Britt, ${ }^{2}$ and Sergi Ferré ${ }^{1}$ \\ ${ }^{1}$ Integrative Neurobiology Section and ${ }^{2}$ Synaptic Plasticity Section, National Institute on Drug Abuse, Intramural Research Program, Baltimore, Maryland \\ 21224
}

It is generally assumed that infralimbic cortex (ILC) and prelimbic cortex, two adjacent areas of the medial prefrontal cortex (mPFC) in rodents, provide selective excitatory glutamatergic inputs to the nucleus accumbens (NAc) shell and core, respectively. It is also generally believed that mPFC influences the extracellular levels of dopamine in the NAc primarily by an excitatory collateral to the ventral tegmental area (VTA). In the present study, we first established the existence of a selective functional connection between ILC and the posteromedial portions of the VTA (pmVTA) and the mNAc shell (pmNAc shell), by measuring striatal neuronal activation (immunohistochemical analysis of ERK1/2 phosphorylation) and glutamate release (in vivo microdialysis) upon ILC electrical stimulation. A novel optogenetic-microdialysis approach allowed the measurement of extracellular concentrations of glutamate and dopamine in the pmNAc shell upon local light-induced stimulation of glutamatergic terminals from ILC. Cortical electrical and local optogenetic stimulation produced significant increases in the extracellular concentrations of glutamate and dopamine in the pmNAc shell. Local blockade of glutamate release by perfusion of an adenosine $\mathrm{A}_{2 \mathrm{~A}}$ receptor antagonist in the pmNAc shell blocked the dopamine release induced by local optogenetic stimulation but only partially antagonized dopamine release induced by cortical electrical stimulation. The results demonstrate that ILC excitatory afferents directly modulate the extracellular concentration of dopamine in the pmNAc shell, but also support the involvement of an indirect mechanism of dopamine control, through a concomitant ILC-mediated activation of the pmVTA.

Key words: dopamine; glutamate; infralimbic cortex; nucleus accumbens; optogenetics; ventral tegmental area

Significance Statement

We established the existence of a functional connection between the infralimbic cortex (ILC) and the posteromedial portions of the ventral tegmental area (pmVTA) and the medial nucleus acumbens shell (pmNAc shell). A novel optogenetic-microdialysis approach allowed us to demonstrate that local glutamate release from glutamatergic terminals from the ILC exert a significant modulation of extracellular concentration of dopamine in the pmNAc shell. This mechanism provides the frame for a selective cortical-mediated tonic dopaminergic modulation of specific striatal compartments.

\section{Introduction}

The monosynaptic glutamatergic pathway that connects the medial prefrontal cortex (mPFC) with the medium spiny efferent neurons of nucleus accumbens (NAc) has received substantial

Received July 28, 2015; revised 0ct. 28, 2015; accepted Nov. 29, 2015.

Author contributions: C.Q., M.O., J.P.B., and S.F. designed research; C.Q., M.O., W.R., A.C.-R., G.Y., and J.P.B. performed research; C.Q., M.O., and S.F. analyzed data; C.Q. and S.F. wrote the paper.

This work supported by the intramural funds of the National Institute on Drug Abuse. We thank Dr Roy A. Wise for his critical review of the paper.

The authors declare no competing financial interests.

Correspondence should be addressed to Dr Sergi Ferré, Integrative Neurobiology Section, National Institute in

Drug Abuse, Intramural Research Program, Triad Technology Building, 333 Cassell Drive, Baltimore, MD 21224. E-mail: sferre@intra.nida.nih.gov.

DOI:10.1523/JNEUROSCI.2850-15.2016

Copyright $\odot 2016$ the authors $\quad 0270-6474 / 16 / 360851-09 \$ 15.00 / 0$ attention due to its distinct involvement in the elicitation of approach (reward-related) and withdrawal (punishment-related) behaviors (Peters et al., 2009; Richard and Berridge, 2013; Moorman et al., 2015). However, it is well known that heterogeneity exists in terms of differential histologic architecture and connectivity in several subterritories of the mPFC. In rodents, most classifications divide the mPFC in (from dorsal to ventral) medial agranular (or medial precentral), anterior cingulate, prelimbic, and infralimbic cortices (Heidbreder and Groenewegen, 2003; Vertes, 2004). Attempts for a functional integration of the cortical subterritories of the mPFC have been made, such as grouping them into a dorsal and a ventral component (Heidbreder and Groenewegen, 2003). However, if anything, a more detailed breakdown of functionally selective areas is required. This becomes more evident when looking at the NAc and its functionally 
different compartments. The anatomically distinct core and shell of the NAc have been seen as functionally distinct dorsal and ventral compartments. It is also generally assumed that the prelimbic cortex (PLC) and the infralimbic cortex (ILC) are the corresponding cortical areas projecting to the NAc core and NAc shell (Peters et al., 2009; Moorman et al., 2015). However, there is clear evidence for a more predominant latero-medial striatal functional compartmentalization, dependent on differential connectivity (Voorn et al., 2004; Ikemoto, 2010). In this way NAc shell can be functionally divided into lateral and medial portions (Voorn et al., 2004; Ikemoto, 2010). It is the medial portion of the NAc shell (mNAc shell) that seems to be preferentially innervated by the ILC and by the posteromedial portion of the ventral tegmental area (pmVTA), which includes the interfascicular nucleus and the central (or caudal) linear nucleus (Ikemoto, 2007). Yet, a clear functional distinction also exists between the anterior and posterior portions of the mNAc shell, with differential involvement in approach and withdrawal behaviors, respectively (Richard and Berridge, 2013; Richard et al., 2013).

The present study was initially aimed at finding a functionalanatomical distinction between the anterior and posterior portions of the mNAc shell. Combining electrical stimulation with ex vivo immunohistochemistry and in vivo microdialysis techniques, we found evidence for a selective connection between ILC, pmVTA, and the posterior portion of the mNAc shell (pmNAc shell). Second, we aimed at studying the mechanisms involved in the cortical-mediated modulation of the extracellular levels of dopamine in the pmNAc shell. Electrical, chemical or transcranial stimulation in different cortical areas has been shown to enhance dopamine release in the corresponding projecting striatal areas (Nieoullon et al., 1978; Murase et al., 1993; Taber et al., 1995; Karreman and Moghaddam, 1996; You et al., 1998; Strafella et al., 2003; Quiroz et al., 2009; Kim et al., 2015). Most of the studies involving cortical stimulation of the mPFC suggested that the concomitant dopamine release in the NAc is mostly dependent on an indirect activation of ventral tegmental area (VTA) dopaminergic neurons (Murase et al., 1993; Taber et al., 1995; Karreman and Moghaddam, 1996; You et al., 1998; Kim et al., 2015). However, there is also compelling evidence, particularly from experiments involving the dorsal striatum, for a local mechanism by which glutamate released by corticostriatal terminals induces striatal dopamine release (Nieoullon et al., 1978; Quarta et al., 2004; Quiroz et al., 2009). With the introduction of a novel microdialysis-optogenetic approach, we obtained evidence for a significant local modulation of extracellular levels of dopamine in the pmNAc by glutamatergic terminals from the ILC, providing a significant mechanism involved in the tonic modulation of dopamine release in this striatal compartment.

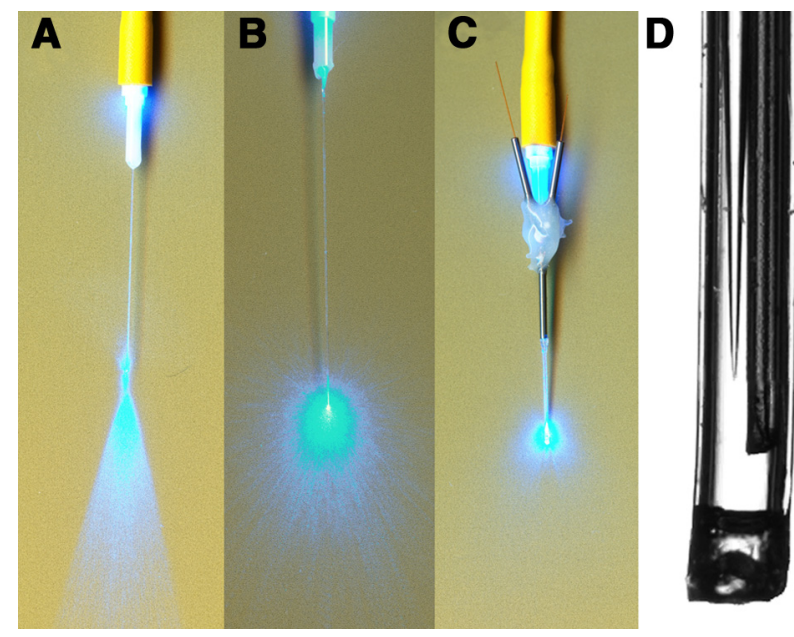

Figure 1. Optogenetic-microdialysis probe. $A$, Light dispersion on a $6 \mu \mathrm{m}$ diamond-coated film from typical optical fiber used for optogenetic stimulation (albeit one-half the diameter). $\boldsymbol{B}$, Light dispersion of the same type of fiber with a conical sculpted tip (see Material and Methods). C, Light dispersion of the fully assembled optogenetic-microdialysis probe. $\boldsymbol{D}$, Magnification of the probe tip, showing the conical sculpted optical fiber and the dialysis inlet tips surrounded by dialysis membrane and epoxy seal at the bottom (see Material and Methods).
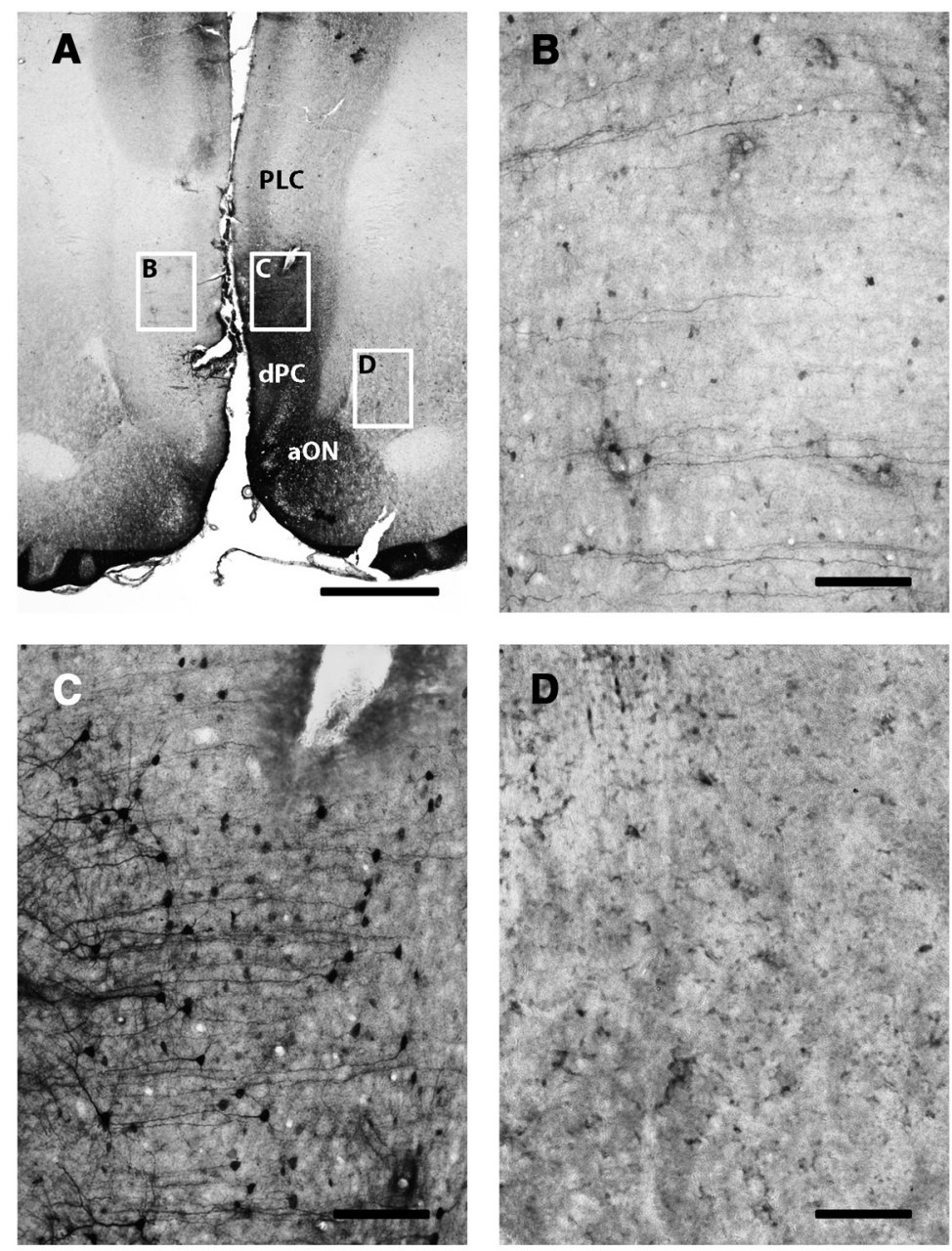

Figure 2. ERK1/2 phosphorylation in the ipsilateral ILC after unilateral electrical stimulation of the ILC. $A$, Immunohistochemical localization of phosphorylated ERK $1 / 2$ in a representative coronal section at $3.0 \mathrm{~mm}$ anterior from bregma; framed areas $B-D$, Correspond to contralateral ILC, ipsilateral ILC, and amNAC shell, respectively; framed area ( shows the track left by one of the tips of the stimulating electrode. Scale bar, $1 \mathrm{~mm}$. $\boldsymbol{B}-\boldsymbol{D}$, Magnification of corresponding framed areas in $\boldsymbol{A}$. Scale bar, $0.1 \mathrm{~mm}$. 

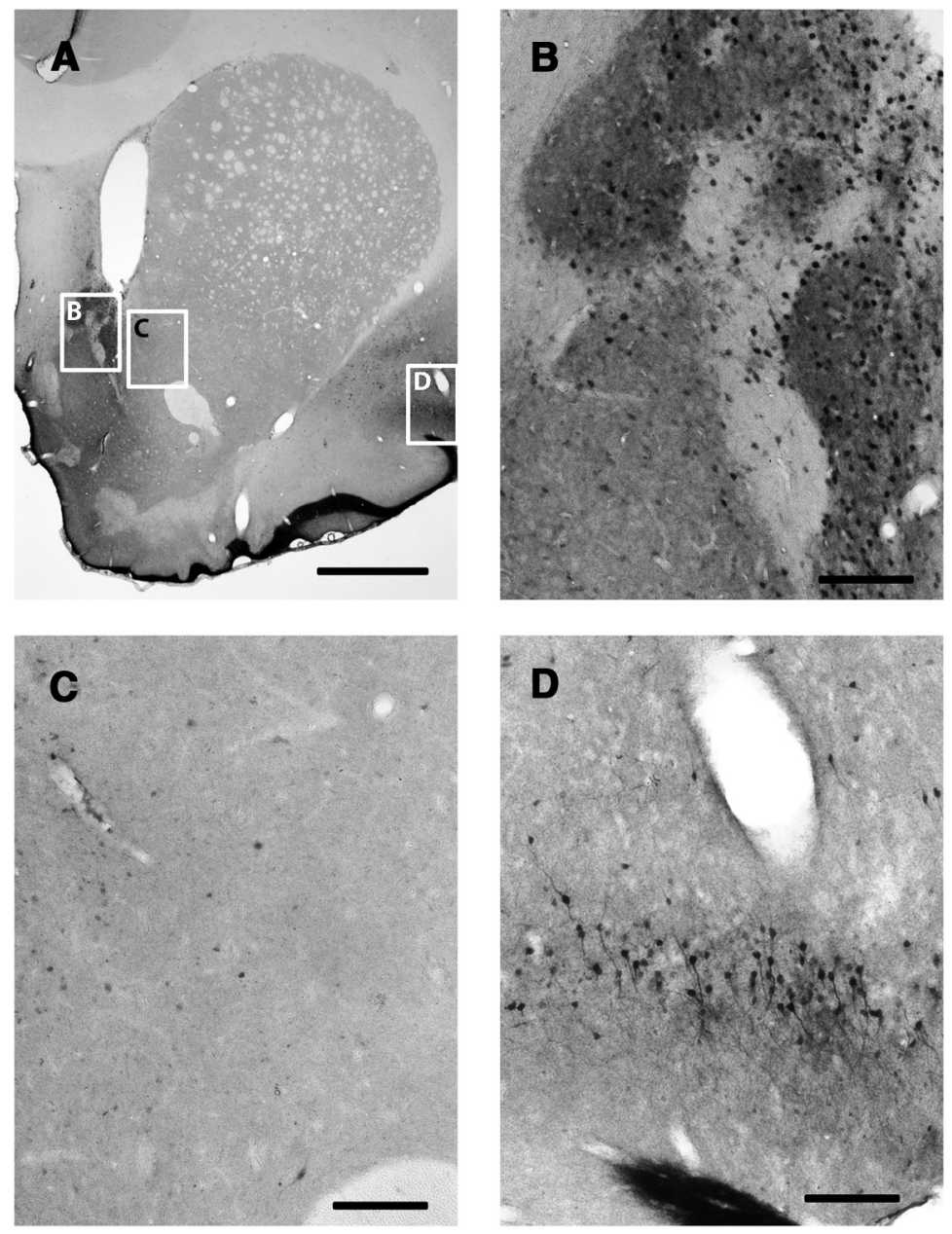

Figure 3. ERK1/2 phosphorylation in the ipsilateral pmNAC shell after unilateral electrical stimulation of the ILC. $A$, Immunohistochemical localization of phosphorylated ERK1/2 in a representative coronal section at $1.5 \mathrm{~mm}$ anterior from bregma; framed areas $B-D$, correspond to ipsilateral pmNAc shell, adjacent NAc core, and AIC, respectively. Scale bar, $1 \mathrm{~mm}$. $\boldsymbol{B}-\boldsymbol{D}$, Magnification of corresponding framed areas in $\boldsymbol{A}$. Scale bar, $0.1 \mathrm{~mm}$.

\section{Materials and Methods}

Animals. Male Sprague-Dawley albino rats (Charles River Laboratories), weighing 300-450 g, were used in all the experimental procedures. Animals were housed two per cage and kept on a $12 \mathrm{~h}$ dark/light cycle with food and water available ad libitum. All animals used in the study were maintained in accordance with the guidelines of the National Institutes of Health Animal Care and the animal research conducted to perform this study was approved by the NIDA IRP Animal Care and Use Committee (protocol no. 12-BNRB-73).

Surgical procedures. For all surgical procedures, animals were anesthetized with $3 \mathrm{ml} / \mathrm{kg}$ of Equithesin (4.44 g chloral hydrate, $0.972 \mathrm{~g} \mathrm{Na}$ pentobarbital, $2.124 \mathrm{~g} \mathrm{MgSO}_{4} 44.4 \mathrm{ml}$ propylene glycol, $12 \mathrm{ml}$ ethanol, and distilled $\mathrm{H}_{2} \mathrm{O}$ up to $100 \mathrm{ml}$ of final solution; NIDA Pharmacy). For intracranial electrical stimulation experiments, animals were implanted unilaterally in the ILC with bipolar tungsten electrodes, $0.12 \mathrm{~mm}$ in diameter and $1 \mathrm{~mm}$ apart (Plastics One); coordinates of implantation were 4.3 and $5.3 \mathrm{~mm}$ anterior, $0.6 \mathrm{~mm}$ lateral, and 5.6 and $5.1 \mathrm{~mm}$ ventral with respect to bregma with a $20^{\circ}$ angle toward the midline. For intracranial electrical stimulation experiments with in vivo microdialysis, concentric microdialysis probes with 2-mm-long dialysis membranes (Eicom) were additionally implanted into the NAc shell ipsilateral to the stimulation electrodes (from 1.2 to $2.5 \mathrm{~mm}$ anterior, $0.5 \mathrm{~mm}$ lateral and $8.0 \mathrm{~mm}$ ventral with respect to bregma). For optogeneticmicrodialysis experiments, animals received unilateral injections of an adeno-associated virus (AAV) encoding Channelrhodopsin 2 (ChR2) fused to enhanced yellow fluorescence protein (EYFP) under control of the CaMKII neuronal promoter (AAVCaMKIIa-hChR2(H134R)-EYFP; University of North Carolina core vector facility) in the ILC; coordinates of injection were $3.0 \mathrm{~mm}$ anterior, $0.5 \mathrm{~mm}$ lateral, and $5.6 \mathrm{~mm}$ ventral with respect to bregma. Virus $(0.5 \mu \mathrm{l}$ of purified and concentrated AAV; $1 \times 10^{12}$ infectious $\mathrm{U} / \mathrm{ml}$ ) was injected using a $105-\mu \mathrm{m}$-thick silica tubing injector coupled directly to a $1 \mu$ l-syringe driven by an infusion pump. Virus suspension was injected over a $10 \mathrm{~min}$ period at a rate of 50 $\mathrm{nl} / \mathrm{min}$ and the injector was left in place for an additional $10 \mathrm{~min}$ to allow diffusion of the suspension. Eight weeks after virus injection, a modified microdialysis probe (optogeneticmicrodialysis probe) with an embedded lightguiding optic fiber (see Intracranial electrical and optogenetic stimulation, below) was implanted into the pmNAc shell; coordinates were $1.2 \mathrm{~mm}$ anterior, $0.5 \mathrm{~mm}$ lateral, and 8.0 $\mathrm{mm}$ ventral with respect to bregma. The electrodes, probes and a head holder (connected to a swivel during stimulation) were fixed to the skull with stainless steel screws and dental acrylic resin.

Intracranial electrical and optogenetic stimulation. For cortical electrical stimulation, the implanted bipolar electrodes were connected to two-coupled constant current isolation units (PSIU6, Grass Instruments) driven by an electrical stimulator (Grass S88; Grass Instruments). Stimulation was applied over a $20 \mathrm{~min}$ period in $160 \mathrm{~ms}$ trains of biphasic rectangular $0.1 \mathrm{~ms}$ pulses of $200 \mu \mathrm{A} 100 \mathrm{~Hz}(1 \mathrm{train} / \mathrm{s})$. These parameters were previously found to be successful at eliciting glutamate release in the dorsal striatum upon cortical stimulation of the lateral agranular motor cortex (Quiroz et al., 2009; Orrú et al., 2011a,b). For optogenetic experiments, a novel optogenetic-microdialysis probe was designed with a $125-\mu \mathrm{m}$ diameter optic fiber ( 0.22 numerical aperture) embedded in a microdialysis probe. The tip of the optic fiber was given a conical shape to allow a local light dispersion (Pisanello et al., 2014) through and around the working portion of the dialyzable membrane (Fig. 1). The conical sculpted tip with the cladding fused to the core was obtained by pulling the fiber with a FlammingBrown pipette puller (Sutter Instruments), fitted with a custom platinum heating filament of circular cross-section ( $1 \mathrm{~mm}$ in diameter) and a holder designed for the small diameter of the optic fiber. Optical stimulation was delivered by coupling the light guiding port of the implanted optogenetic-microdialysis probe to a $473 \mathrm{~nm}$ solid-state laser module driven by the electrical stimulator (Grass S88 stimulator). Light was applied over a $20 \mathrm{~min}$ period in $160 \mathrm{~ms}$ trains of $1 \mathrm{~ms}$ pulses at $100 \mathrm{~Hz}$ at $5-8$ $\mathrm{mW}$ at the probe tip ( 1 train/s). Light intensity at the probe tip was measured using an integrating sphere silicon photodiode power sensor designed for optical power measurements independent of beam shape and divergence (model S144C, Thor Labs).

In vivo microdialysis. Microdialysis sampling was performed during electrical or optogenetic stimulation to analyze the extracellular concentrations of glutamate and dopamine in the mNAc shell of freely moving rats $24 \mathrm{~h}$ after probe implantation. Striatal adenosine $\mathrm{A}_{2 \mathrm{~A}}$ receptors localized in glutamatergic terminals exert a strong modulatory role of glutamate release (Quarta et al., 2004) and systemic administration or local perfusion of $\mathrm{A}_{2 \mathrm{~A}}$ receptor antagonists have been shown to block glutamate release in the dorsal striatum induced by electrical stimulation in the lateral agranular motor cortex (Quiroz et al., 2009; Orrú et al., $2011 \mathrm{a}, \mathrm{b})$. Local perfusion in the pmNAc shell of the $\mathrm{A}_{2 \mathrm{~A}}$ receptor antagonist MSX-3 was then used to evaluate the role of glutamate on dopa- 

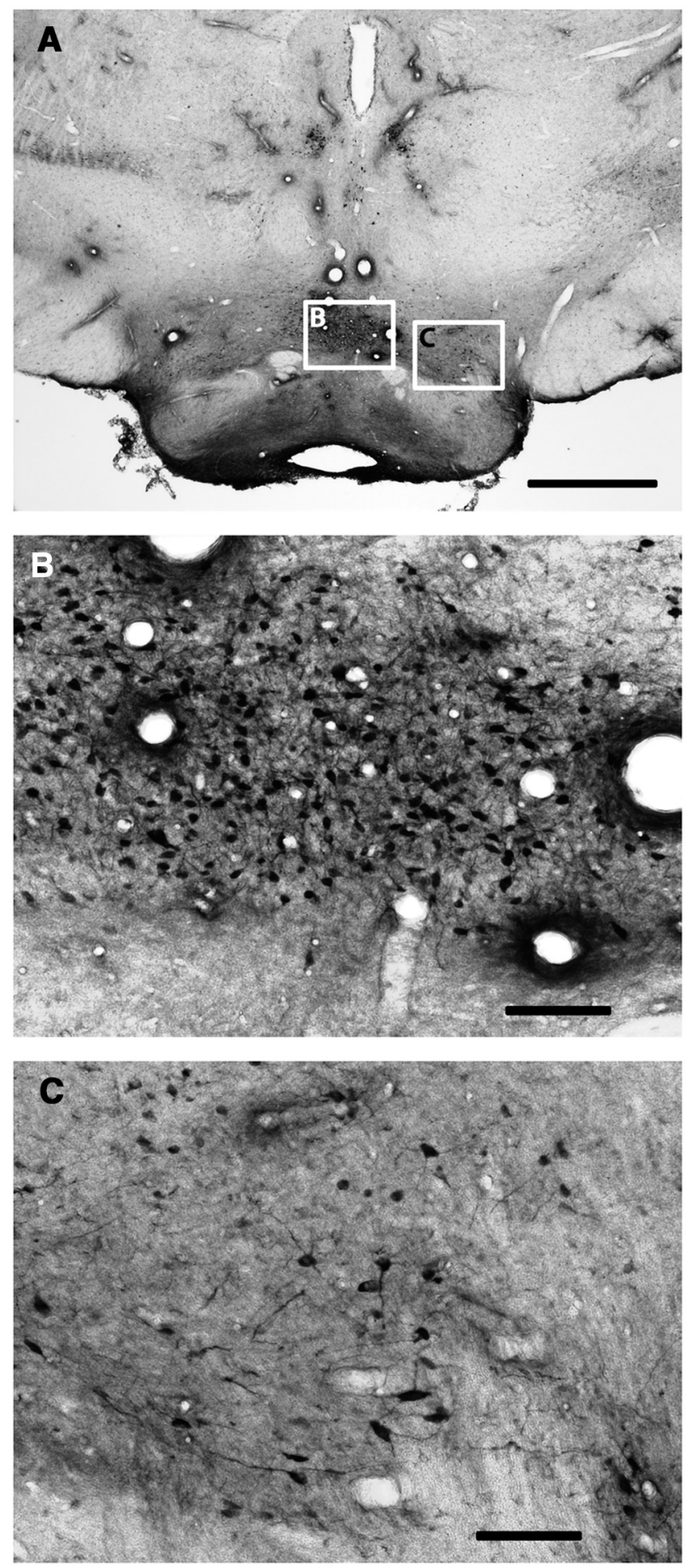

Figure 4. ERK $1 / 2$ phosphorylation in the pmVTA after unilateral electrical stimulation of the ILC. $A$, Immunohistochemical localization of phosphorylated ERK1/2 in a representative coronal section at $6.4 \mathrm{~mm}$ posterior from bregma. BA, Basilar artery. Scale bar, $1 \mathrm{~mm}$. B, C, Magnification of corresponding framed areas in $A$. cLN, Caudal linear nucleus; IFN, interfascicular nucleus; IPN, interpeduncular nucleus. Scale bar, $0.1 \mathrm{~mm}$.

mine release upon ILC electrical or pmNAc optogenetic stimulation. An artificial cerebrospinal solution [aCSF; containing the following (in $\mathrm{mM}$ ) $144 \mathrm{NaCl}, 4.8 \mathrm{KCl}, 1.7 \mathrm{CaCl}_{2}$, and $1.2 \mathrm{MgCl}_{2}$ ] was initially perfused for $120 \mathrm{~min}$ through the microdialysis or the optogenetic-microdialysis probe at a constant rate of $1 \mu \mathrm{l} / \mathrm{min}$ and subsequently switched (or not) to aCSF + MSX-3 $(1 \mu \mathrm{M})$ for 120 more minutes before cortical or optogenetic stimulation. Sampling was obtained at $20 \mathrm{~min}$ intervals. Electrical or optogenetic stimulation was applied for $20 \mathrm{~min}$, and $20 \mathrm{~min}$ samples were taken for an additional $80 \mathrm{~min}$ after the beginning of the stimulation. Glutamate content was measured by HPLC coupled to a glutamate oxidase enzyme reactor and electrochemical detector (Eicom). Dopamine was measured by HPLC coupled with a coulometric detector (5200a Coulochem III, ESA). At the end of the microdialysis experiment or after the stimulation experiments without microdialysis, animals were deeply anesthetized with Equithesin and perfused transcardially with 0.1 м PBS, pH 7.4, followed by $4 \%$ formaldehyde in $0.1 \mathrm{~m}$ PBS. Brains were kept in the same fixative for $2 \mathrm{~h}$ and then stored in $30 \%$ sucrose $/ 0.1 \mathrm{M}$ PBS solution for $48 \mathrm{~h}$ at $4^{\circ} \mathrm{C}$. Forty micrometer coronal sections were cut in a Leica CM $3050 \mathrm{~S}$ cryostat at $-20^{\circ} \mathrm{C}$, collected in PBS, and stored in antifreeze-buffered solution (20\% ethylene glycol, $10 \%$ glycerol, and $10 \%$ sucrose in PBS) at $-80^{\circ} \mathrm{C}$ until processing. Sections were then evaluated for localization of implanted electrodes and probes and ChR2EYFP expression. Confocal fluorescence microscopy images were acquired with a Zeiss microscope (Examiner Z1) fitted with a confocal laser module (LSM-710, Zeiss).

ERK1/2 immunohistochemistry. Immediately after the offset of cortical stimulation, rats were deeply anesthetized, perfused transcardially, and the brains processed and sectioned as indicated above. The slices were then rinsed with PBS, incubated with $50 \mathrm{~mm}$ ammonium chloride (in PBS) for $10 \mathrm{~min}$ and $0.1 \%$ hydrogen peroxide in PBS for $10 \mathrm{~min}$, rinsed, and incubated in blocking buffer (PBS/0.1\% Triton X-100/5\% bovine serum albumin) for $2 \mathrm{~h}$ before incubation with rabbit polyclonal antiphospho-Thr202/Tyr204 ERK1/2 (1:2000 dilution; Cell Signaling Technology) in blocking buffer for $24 \mathrm{~h}$ at $4^{\circ} \mathrm{C}$. Sections were washed and incubated in 1:200 biotinylated goat anti-rabbit antibody (Vector Laboratories) for $2 \mathrm{~h}$, then washed and incubated for $2 \mathrm{~h}$ in $\mathrm{ABC}$ reagent (PK-6100; Vector Laboratories), washed again, treated with $0.33 \mathrm{mg} / \mathrm{ml}$ 3,3-diaminobenzidine, $50 \mathrm{~mm}$ ammonium nickel sulfate, and $0.003 \%$ hydrogen peroxide solution in PBS until development (5-10 min), transferred to PBS, mounted onto chromalum gelatin-coated slides, air-dried, dehydrated through graded ethanol solutions, cleared in xylene, and coverslipped with DPX (mixture of xylene and dibutyl phthalate; Fisher Scientific). Images were acquired using a digital camera (HRC; Zeiss) coupled to an Axioimager A1 microscope (Zeiss). For quantification of cells positive for ERK1/2 phosphorylation, $40-\mu \mathrm{m}$-thick sections where stained as described above and images where acquired with the Axioimager A1 microscope with the following parameters: $20 \times$ magnification, 0.3 of numerical aperture, $3200 \mathrm{~K}$ of color temperature illumination, 1 ms of camera exposure time and $1040 \times 1388$ pixel resolution for a field of $517 \times 691 \mu \mathrm{m}$. Quantified areas correspond to the framed fields in Figures 2-4. Stained cell somas were manually counted using the cell counter utility of the ImageJ 1.49 image analysis software (National Institutes of Health).

\section{Results}

Neuronal activation in the pmNAc and pmVTA induced by electrical stimulation of the ILC

ERK1/2 phosphorylation was examined as an indicator of postsynaptic activation of functional connectivity with the ILC. As shown in Figure 2 (3.0 $\mathrm{mm}$ anterior from bregma), electrical stimulation in the ILC induced ERK1/2 phosphorylation in the ipsilateral, but not contralateral ILC (Fig. 2C,B, respectively). ERK1/2 phosphorylation extended ventrally to the dorsal peduncular cortex (dPC) and anterior olfactory nucleus (aON; which also showed some contralateral activation), but not to the anterior portion of the medial NAc shell (amNAc shell; Fig. 2D) and did not extent dorsally to the PLC. Analysis of serial coronal sections demonstrated that ERK1/2 phosphorylation spanned the whole anterior-posterior length of the ILC (from 3.7 to 2.7 $\mathrm{mm}$ anterior from bregma). As shown in Figure 3, at $1.5 \mathrm{~mm}$ anterior from bregma, ILC stimulation induced ipsilateral ERK1/2 phosphorylation in the mNAc shell (Fig. $3 B$ ), but not in the adjacent NAc core (Fig. $3 C$ ). Some activation was also evident in the agranular insular cortex (AIC; Fig. 3D). ERK1/2 phosphor- 
A

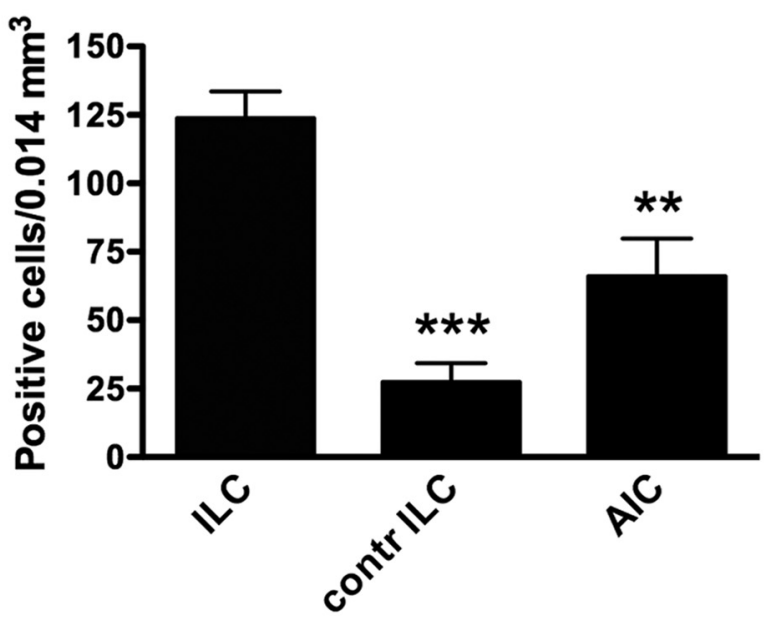

B

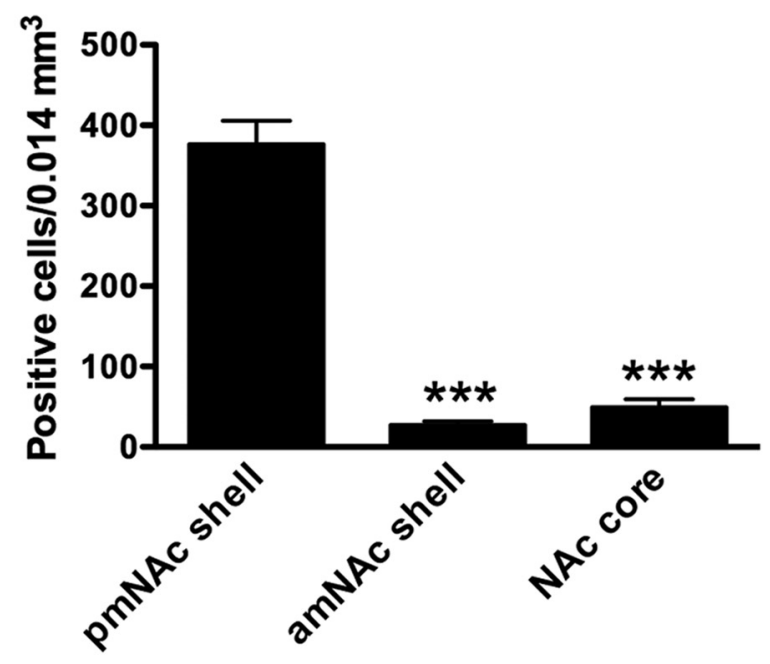

C

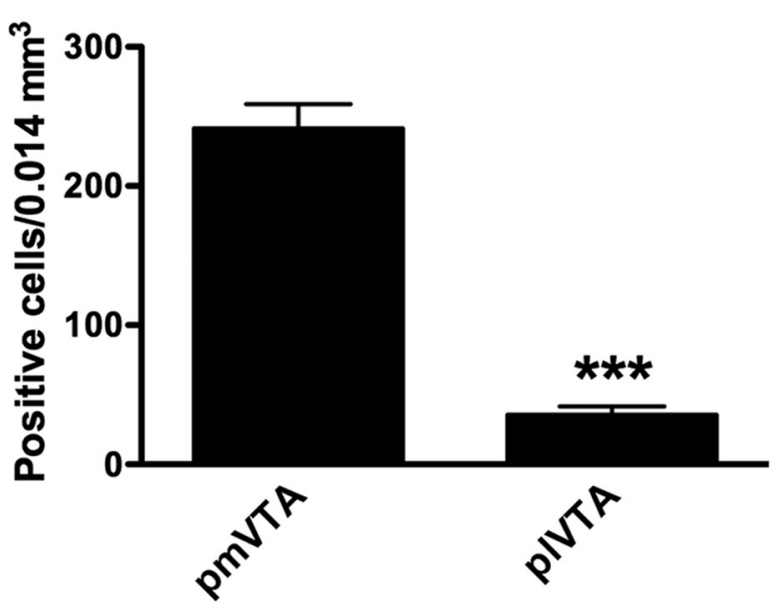

Figure 5. Quantification of positive cells stained for ERK1/2 phosphorylation in cortical, striatal and mesencephalic areas after stimulation of the ILC. $A$, Number of positive cells in ipsilateral and contralateral (contr) ILC (Fig. 2B, $C$ and AIC (Fig. 3D). B, Number of positive cells in pmNAc and amNAc shell (Figs. 3B, 2A) and NAc core (Fig. 3C). C, Number of positive cells in pmVTA and pIVTA (Fig. 4B, C). Results are expressed as means \pm SEM $(n=5)$. ${ }^{* *} p<0.001$ compared with ILC (in $\boldsymbol{A}$ ), pmNAc shell (in $\boldsymbol{B}$ ) or pmVTA (in $\boldsymbol{C}$ ); repeated-measures ANOVA followed by Newman-Keuls test or paired $t$ test). ylation was only evident in the pmNAc shell, and was not observed in sections more rostral than 1.8 anterior to bregma (Fig. $2 D$; see absence of activation of the amNAc shell). As shown in Figure 4, at $6.4 \mathrm{~mm}$ posterior from bregma, ILC stimulation induced ERK1/2 phosphorylation in the pmVTA (Fig. $4 B$; corresponding to the interfascicular nucleus and caudal linear nucleus in the atlas of Paxinos and Watson, 2007). Sparse labeling could also be observed laterally to the pmVTA (Fig. 4C; corresponding to parabrachial and paranigral VTA; Paxinos and Watson, 2007). These results of functional connectivity with the ILC showed a very similar pattern to the anatomical study by Vertes (2004; see Discussion), including the connectivity with the AIC and additional connectivity with septal, amygdalar, thalamic, and hypothalamic areas that were not the object of the present study. Figure 5 shows the average of quantification of positive cells from the framed fields shown in Figures 2-4 for several animals $(n=5)$. Results from ipsilateral versus contralateral ILC, pmNAc shell versus adjacent NAc core and versus amNAC shell and medial versus lateral VTA were found to be significantly different (repeated-measures ANOVA followed by Newman-Keuls test or Student's paired $t$ test: $p<0.001$ in all cases).

Glutamate and dopamine release in the pmNAc shell induced by electrical stimulation in the ILC

Electrical stimulation in the ILC produced a significant increase in the extracellular concentrations of glutamate (up to $\sim 300 \%$ of basal levels; repeated-measures ANOVA followed by NewmanKeuls test; $p<0.01$ ) and dopamine (up to $\sim 250 \%$ of basal levels; repeated-measures ANOVA; $p<0.01$ ) in the mNAc (Fig. $6 B, C$ ). These effects were always observed when the dialysis probes were located between 1.2 and $1.5 \mathrm{~mm}$ anterior from bregma; negative results were always obtained with probes located at more anterior than $1.8 \mathrm{~mm}$ with respect to bregma (Fig. $6 A, B, C$ ). Mixed results were obtained when the probe was located at $\sim 1.8 \mathrm{~mm}$ (Fig. $6 A$ ), the anterior border of the ILC-innervated pmNAc shell according to the results of ERK1/2 phosphorylation. The distance of 1.2 $\mathrm{mm}$ anterior to bregma was chosen for subsequent microdialysis experiments. Constant perfusion of MSX-3 (1 $\mu \mathrm{M})$ through the microdialysis probe (reverse dialysis) did not modify the basal striatal extracellular concentrations of glutamate or dopamine, as analyzed by comparing the average values of the samples prior and during MSX-3 perfusion before cortical or optogenetic stimulation. Average values for glutamate before and after MSX-3 were $1.1 \pm 0.2$ and $0.9 \pm 0.2 \mu \mathrm{M}$, respectively $(n=26 ; p>0.05$; paired $t$ test). Average values for dopamine before and after MSX-3 were $2.7 \pm 0.3$ and $2.2 \pm 0.3 \mathrm{nM}$, respectively $(n=23 ; p>$ 0.05 ; paired $t$ test). However, in the presence of MSX-3 (1 $\mu \mathrm{M})$, ILC stimulation did not produce a significant increase in the extracellular concentration of glutamate in the pmNAc shell (Fig. $6 B$ ), although it produced a significantly attenuated (but not completely antagonized, up to $\sim 150 \%$ of basal levels; repeatedmeasures ANOVA followed by Newman-Keuls test; $p<0.05$ ) dopamine response (Fig. 6C).

Glutamate and dopamine release in the pmNAc shell induced by optogenetic stimulation of terminals from a direct ILC projection to the pmNAC shell

The microdialysis experiments after cortical electrical stimulation demonstrated that $\mathrm{A}_{2 \mathrm{~A}}$ receptors in the pmNAc shell exert a strong local control of glutamate release evoked by ILC stimulation. They also suggested that a component of the dopamine release in the pmNAC shell induced by ICL stimulation was secondary to local glutamate release. An optogenetic-microdialysis 
probe was designed to further analyze this possibility and allowed the measurement of extracellular concentrations of glutamate and dopamine in the pmNAC shell upon direct light-induced stimulation of glutamatergic terminals expressing the light-activated cation channel ChR2. An optical fiber was introduced in a microdialysis probe and its tip was modified to allow local laser delivery around the dialysis membrane (Fig. 1; for more details, see Materials and Methods). Eight weeks after microinjection in the ILC of an AAV encoding ChR2-EYFP under control of the CaMKII neuronal promoter a unilateral expression of ChR2-EYFP in the ILC (Fig. 7A) and in the pmNAc shell (Fig. $8 A, B, D)$, but not the adjacent NAc core (Fig. $8 A, C, E$ ) or the amNAc shell (Fig. $7 B$ ), could be observed by confocal microscopy (in addition to other ILC projecting brain areas, such as septal, amygdalar, hypothalamic, and thalamic areas, which were not the object of the present study). After the optogeneticmicrodialysis probe was implanted at 1.2 $\mathrm{mm}$ anterior to bregma, optogenetic stimulation (with the same frequency and duration as that applied with electrical stimulation: $100 \mathrm{~Hz}$ and $20 \mathrm{~min}$ ) produced a very similar and significant increase in the extracellular concentration of glutamate as compared with that induced by ILC electrical stimulation, $\sim 300 \%$ of basal levels (repeated-measures ANOVA followed by Newman-Keuls test; $p<0.01$ ) in the pmNAc shell (Fig. 9A). Dopamine, on the other hand, only increased to $150 \%$ (repeated-measures ANOVA; $p<0.01$; Fig. 9B), which was a similar increase to that observed with ILC stimulation in the presence of the $\mathrm{A}_{2 \mathrm{~A}}$ receptor antagonist MSX-3 (Fig. 6C). Different from the experiments with cortical electrical stimulation, perfusion with MSX-3 completely counteracted not only glutamate but also dopamine release induced by optogenetic stimulation (Fig. 9A,B).

\section{Discussion}

The present study first demonstrates the existence of a restricted functional connection between the rat ILC and the pmNAc but not amNAc shell. This is supported by previous anatomical studies by Vertes (2004). By using an anterograde tracing technique, he described that PLC fibers distribute massively throughout the core and shell regions, but in contrast, ILC fibers project fairly selectively to the caudal mNAc shell (Vertes, 2004). This portion of the shell may have significance for reward function. Thompson and Swanson (2010) conducted a more restricted, multiple and serial anterograde-retrograde anatomical connectivity analysis starting in the mNAc shell, aiming at the distinct portion of the rat anterior mNAc shell area where microinjection of opioids strongly potentiates a behavioral orofacial response elicited by sucrose taste (Peciña and Berridge, 2005). They found a very selective connection of the mNAC shell with the ILC and also with other brain areas, including the pmVTA (Thompson and Swanson, 2010), which is the main source of the dopaminergic input to the most medial striatal areas (mNAc shell and medial
B

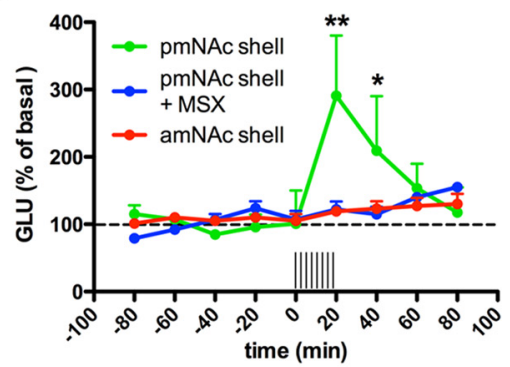

C

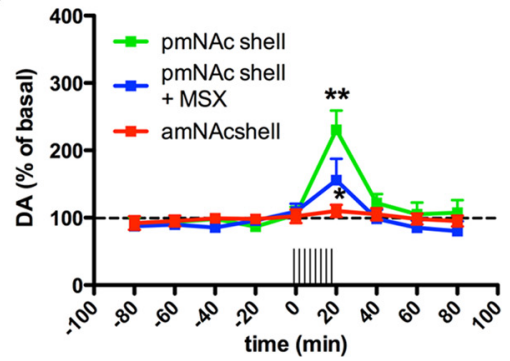

Figure 6. Effect of unilateral electrical stimulation in the ILC on the extracellular levels of glutamate and dopamine in the mNAc

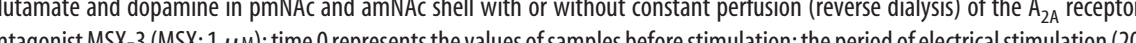
$\mathrm{min}$ ) is represented as a train of vertical lines; results are expressed as mean $\pm \mathrm{SEM}$ of percentage of the average of six values before stimulation ( $n=6-10$ per group); ${ }^{*} p<0.05,{ }^{* *} p<0.01$, compared with value of the last sample before the stimulation, respectively (repeated-measures ANOVA followed by Newman-Keuls test).

olfactory tubercle; Ikemoto, 2007, 2010). In the present study, by analyzing P-ERK1/2-induced neuronal activation upon ILC stimulation, we found an additional selective functional connection between the ILC and the pmVTA, highlighting the circuitry that interconnects the three brain areas: ILC, pmVTA, and pmNAc shell (Fig. 8).

The anterior part of the mNAc shell (amNAc shell) is involved with approach, appetitive, reward-related behaviors, whereas the pmNAc shell is involved with withdrawal and threat-related behaviors. This functional distinction has been largely supported by experiments with local injections (for review, see Richard et al., 2013). Also, several lines of research point to a very salient role of a brain circuit including the ILC and the amygdala in the suppression of threat conditioning (Vidal-Gonzalez et al., 2006; Knapska et al., 2012; Moscarello and LeDoux, 2013). According to the present study, the ILC innervates the whole withdrawal-involved area of the mNAc shell and the restricted opioid-sensitive, appetitive-related behavior portion of the mNAc shell. In fact, neurochemical activation of the ILC was recently shown to suppress both appetitive and threat-related behaviors generated by selective disruptions of glutamate transmission in the anterior and posterior mNAc shell, respectively (Richard and Berridge, 2013). Therefore, the established "anatomical" separation between PLC and ILC could be revised to a more functional demarcation and be moved more ventrally to accommodate the cortical inputs to the functionally divided anterior, approach-involved, and posterior, withdrawal-involved, halves of the mNAc shell.

As mentioned in the Introduction, it is generally believed that mPFC influences the extracellular levels of dopamine in the NAc primarily by an excitatory collateral to the VTA. However, previous studies have found evidence for a local impulse-independent 

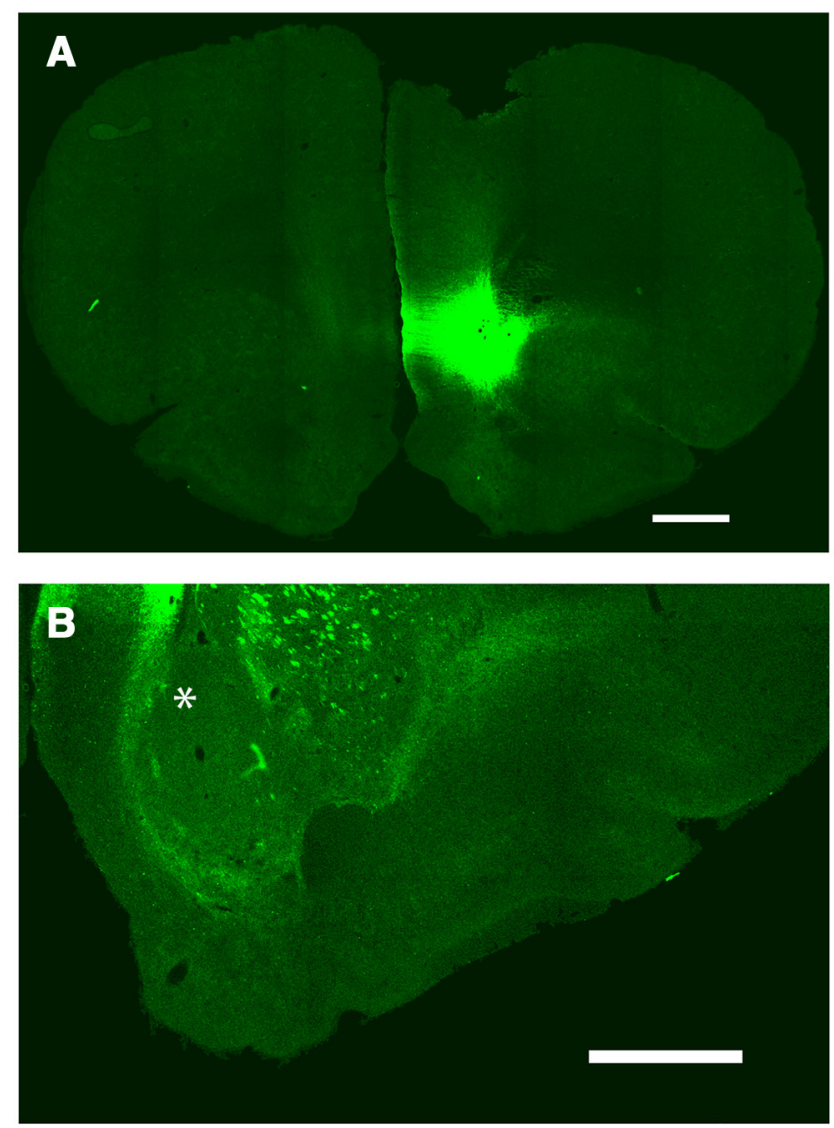

Figure 7. Confocal laser microscopy of coronal brain sections showing the localization of ChR2-EYFP 8 weeks after unilateral AAV microinjection in the ILC. $A$, Unilateral expression of ChR2-EYFP in the ILC; coronal section at $3.0 \mathrm{~mm}$ anterior from bregma. Scale bar, $1 \mathrm{~mm}$. $\boldsymbol{B}$, Lack of expression of ChR2-EYFP in the ipsilateral amNAc shell (asterisk; coronal section at $2.3 \mathrm{~mm}$ anterior from bregma; scale bar, $1 \mathrm{~mm}$ ).

and NMDA receptor- and dopamine transporter-dependent mechanism that modulates striatal dopamine release (for review, see Leviel, 2011). Through this mechanism, activation of extrasynaptic NMDA receptors (and possibly also AMPA and metabotropic glutamate receptors) localized in dopaminergic terminals leads to dopamine release by reversal of the dopamine transporter (Leviel, 2011). The "striatal spine module" has been invoked as a model that provides the anatomical frame for this local glutamate-mediated modulation of dopamine release (Ferré et al., 2007, 2009). This functional module comprises the dendritic spine of the striatal medium spiny neuron, the glutamatergic and dopaminergic terminals that make synaptic contact with the head and neck of the dendritic spine, respectively, and astroglial processes that wrap around the glutamatergic synapse. In view of the key role of astrocytes on the modulation of the relatively high extracellular levels of glutamate (Baker et al., 2002), it is improbable that endogenous glutamate spilling over from the glutamatergic synapse is directly responsible for the stimulation of NMDA receptors localized in the dopaminergic terminal. A more plausible hypothesis is that these receptors are under the control of astrocytic glutamate release, which depends on neuronal glutamate release (Fields and Stevens-Graham, 2002, Ferré et al., 2007). Factors that modulate extracellular glutamate therefore appear to play an indirect role on the modulation of tonic dopamine. The present study demonstrates that adenosine, acting on $\mathrm{A}_{2 \mathrm{~A}}$ receptors localized in corticostriatal glutamatergic terminals, constitutes a particularly significant factor, because local $\mathrm{A}_{2 \mathrm{~A}}$ re-
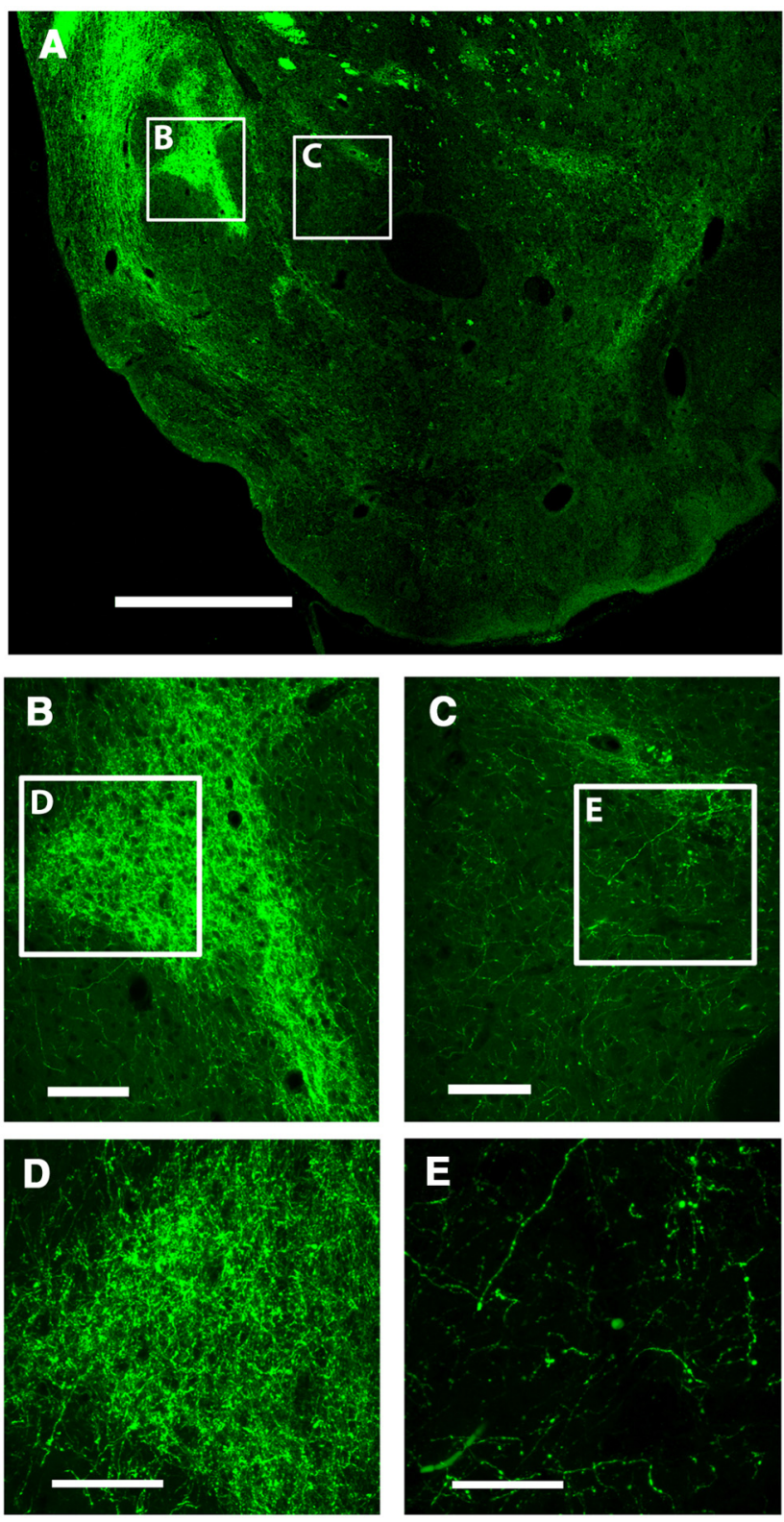

Figure 8. Confocal laser microscopy of coronal brain sections showing the localization of ChR2-EYFP 8 weeks after unilateral AAV microinjection in the ILC. $A$, Expression of ChR2-EYFP in a coronal section at $1.2 \mathrm{~mm}$ anterior from bregma; framed areas $B$ and $C$ correspond to pmNAC shell and adjacent NAc core, respectively. Scale bar, $1 \mathrm{~mm}$. B, C, Magnification of corresponding framed areas in $\boldsymbol{A}$. Scale bar, $0.2 \mathrm{~mm}$. D, E, Magnification of corresponding framed areas in $\boldsymbol{B}$ and C showing ICL nerve terminals. Scale bar, $0.2 \mathrm{~mm}$.

ceptor blockade (by striatal perfusion of a selective antagonist) totally abrogated the local glutamate and dopamine release induced by optogenetic stimulation.

On the other hand, although the $\mathrm{A}_{2 \mathrm{~A}}$ receptor antagonist also counteracted glutamate release in the pmNAC shell induced by ILC electrical stimulation, it only partially attenuated dopamine release. Adenosine $\mathrm{A}_{2 \mathrm{~A}}$ receptors are known potent modulators of local striatal glutamatergic neurotransmission and are localized in corticostriatal glutamatergic terminals but not in striatal dopaminergic terminals (Quiroz et al., 2009; Orrú et al., $2011 \mathrm{a}, \mathrm{b})$. The counteracting effect of the $\mathrm{A}_{2 \mathrm{~A}}$ receptor antagonist on glutamate and dopamine release induced by cortical electrical or striatal optogenetic stimulation cannot be related to a lowering effect of MSX-3 on the basal levels of either neurotransmitter. We 
A

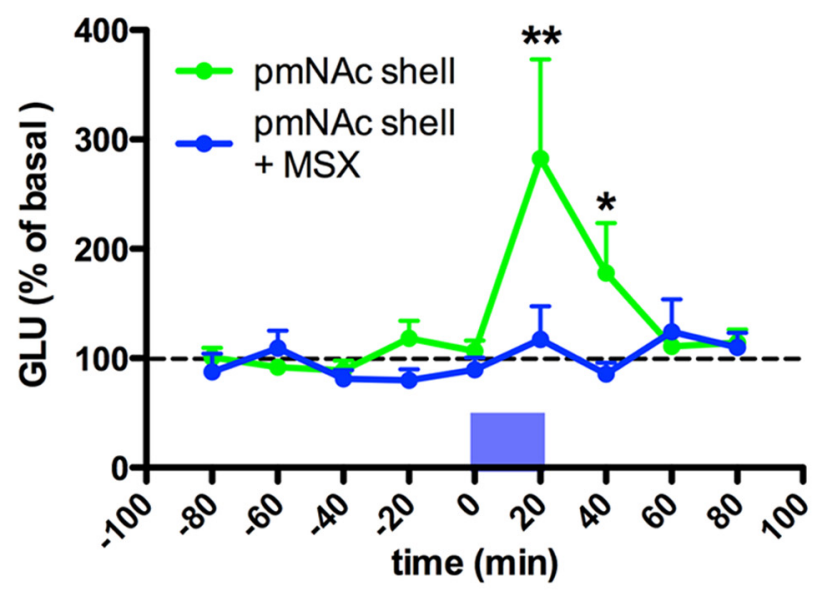

B

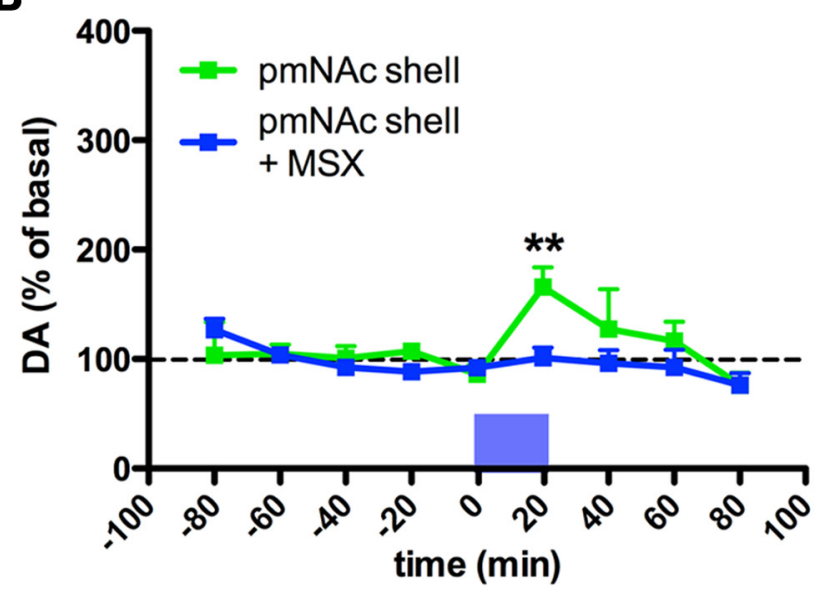

Figure 9. Effect of optogenetic stimulation in the pmNAc shell. $\boldsymbol{A}, \boldsymbol{B}$, Time course of extracellular concentrations of glutamate and dopamine in pmNAc shell with or without constant perfusion (reverse dialysis) of the $A_{2 A}$ receptor antagonist MSX-3 (MSX; $1 \mu \mathrm{M}$ ); time 0 represents the values of samples before stimulation; the period of optogenetic stimulation ( $20 \mathrm{~min}$ ) is represented as a blue bar; results are expressed as mean \pm SEM of percentage of the average of six values before stimulation ( $n=6-10$ per group). ${ }^{*} p<0.05,{ }^{* *} p<0.01$ compared with value of the last sample before the stimulation, respectively (repeated-measures ANOVA followed by Newman-Keuls test).

have previously shown that perfusion the $\mathrm{A}_{2 \mathrm{~A}}$ receptor antagonist MSX-3 in the NAc shell (using the same concentration, $1 \mu \mathrm{M}$, and the same rat strain and microdialysis methodology) does not produces a significant change in the striatal extracellular concentration of glutamate or dopamine, although it completely counteracts the significant increases of both neurotransmitters $(\sim 250$ and $150 \%$ of basal levels, respectively) induced by the $\mathrm{A}_{2 \mathrm{~A}}$ receptor agonist CGS 21680 (10 $\mu \mathrm{M}$; Quarta et al., 2004). In agreement, basal levels of extracellular glutamate or dopamine were not modified by perfusion with MSX-3 in the present experiment. Therefore, the present results indicate that the ILC controls the extracellular levels of dopamine in the pmNAc shell by means of both the direct ILC-pmNAc pathway and the indirect ILCpmVTA-pmNAc pathway. Recent morphological and functional in vitro studies have found evidence for a small percentage of NAc dopaminergic terminals that are able to corelease dopamine and glutamate (Stuber et al., 2010; Zhang et al., 2015). Nevertheless, the significant dopamine release in the absence of glutamate release observed in the pmNAc shell upon local perfusion of the $\mathrm{A}_{2 \mathrm{~A}}$ receptor antagonist during ILC stimulation suggests that, in vivo, the indirect ILC-pmVTA-pmNAc pathway does not contribute strongly to the extracellular levels of glutamate in the mNAc shell.

Basically two different temporal operating modes of dopamine neuronal function have been described: a fast, millisecondscale, phasic response, which codes for a reward prediction error (Schultz, 2002), and a prolonged, minute-scale, and tonic dopamine modulation (Schultz, 2002), recently suggested to provide signals of proximity and value of distant rewards (Howe et al., 2013). Although dopaminergic cell firing is involved in the phasic and also in the tonic response, the present study underscores a role of local glutamate release by corticostriatal terminals in the modulation of the tonic striatal extracellular levels of dopamine, at least in ILC-innervated pmNAc shell.

In summary, the present study demonstrates that a glutamatergic innervation of the ILC directly modulates the extracellular concentration of dopamine in the pmNAc shell (direct mechanism). The study also indicates the involvement of an indirect mechanism, through a concomitant ILC-mediated activation of the pmVTA. The direct mechanism provides the frame for a selective cortical-mediated tonic dopaminergic modulation of specific striatal compartments. However, the role of the direct and the indirect mechanisms in other striatal compartments needs still to be determined.

\section{References}

Baker DA, Xi ZX, Shen H, Swanson CJ, Kalivas PW (2002) The origin and neuronal function of in vivo nonsynaptic glutamate. J Neurosci 22: 9134-9141. Medline

Ferré S, Agnati LF, Ciruela F, Lluis C, Woods AS, Fuxe K, Franco R (2007) Neurotransmitter receptor heteromers and their integrative role in "local modules": the striatal spine module. Brain Res Rev 55:55-67. CrossRef Medline

Ferré S, Goldberg SR, Lluis C, Franco R (2009) Looking for the role of cannabinoid receptor heteromers in striatal function. Neuropharmacology 56:226-234. CrossRef Medline

Fields RD, Stevens-Graham B (2002) New insights into neuron-glia communication. Science 298:556-562. CrossRef Medline

Heidbreder CA, Groenewegen HJ (2003) The medial prefrontal cortex in the rat: evidence for a dorso-ventral distinction based upon functional and anatomical characteristics. Neurosci Biobehav Rev 27:555-579. CrossRef Medline

Howe MW, Tierney PL, Sandberg SG, Phillips PE, Graybiel AM (2013) Prolonged dopamine signalling in striatum signals proximity and value of distant rewards. Nature 500:575-579. CrossRef Medline

Ikemoto S (2007) Dopamine reward circuitry: two projection systems from the ventral midbrain to the nucleus accumbens-olfactory tubercle complex. Brain Res Rev 56:27-78. CrossRef Medline

Ikemoto S (2010) Brain reward circuitry beyond the mesolimbic dopamine system: a neurobiological theory. Neurosci Biobehav Rev 35:129-150. CrossRef Medline

Karreman M, Moghaddam B (1996) The prefrontal cortex regulates the basal release of dopamine in the limbic striatum: an effect mediated by ventral tegmental area. J Neurochem 66:589-598. CrossRef Medline

Kim IH, Rossi MA, Aryal DK, Racz B, Kim N, Uezu A, Wang F, Wetsel WC, Weinberg RJ, Yin H, Soderling SH (2015) Spine pruning drives antipsychotic-sensitive locomotion via circuit control of striatal dopamine. Nat Neurosci 18:883-891. CrossRef Medline

Knapska E, Macias M, Mikosz M, Nowak A, Owczarek D, Wawrzyniak M, Pieprzyk M, Cymerman IA, Werka T, Sheng M, Maren S, Jaworski J, Kaczmarek L (2012) Functional anatomy of neural circuits regulating fear and extinction. Proc Natl Acad Sci U S A 109:17093-17098. CrossRef Medline

Leviel V (2011) Dopamine release mediated by the dopamine transporter, facts and consequences. J Neurochem 118:475-489. CrossRef Medline

Moorman DE, James MH, McGlinchey EM, Aston-Jones G (2015) Differential roles of medial prefrontal subregions in the regulation of drug seeking. Brain Res 1628:130-146. CrossRef Medline

Moscarello JM, LeDoux JE (2013) Active avoidance learning requires pre- 
frontal suppression of amygdala-mediated defensive reactions. J Neurosci 33:3815-3823. CrossRef Medline

Murase S, Grenhoff J, Chouvet G, Gonon FG, Svensson TH (1993) Prefrontal cortex regulates burst firing and transmitter release in rat mesolimbic dopamine neurons studied in vivo. Neurosci Lett 157:53-56. CrossRef Medline

Nieoullon A, Cheramy A, Glowinski J (1978) Release of dopamine evoked by electrical stimulation of the motor and visual areas of the cerebral cortex in both caudate nuclei and in the substantia nigra in the cat. Brain Res 145:69-83. CrossRef Medline

Orrú M, Quiroz C, Guitart X, Ferré S (2011a) Pharmacological evidence for different populations of postsynaptic adenosine $\mathrm{A} 2 \mathrm{~A}$ receptors in the rat striatum. Neuropharmacology 61:967-974. CrossRef Medline

Orrú M, Bakešová J, Brugarolas M, Quiroz C, Beaumont V, Goldberg SR, Lluís C, Cortés A, Franco R, Casadó V, Canela EI, Ferré S (2011b) Striatal pre- and postsynaptic profile of adenosine $\mathrm{A}(2 \mathrm{~A})$ receptor antagonists. PLoS One 6:e16088. CrossRef Medline

Paxinos G, Watson C (2007) The rat brain in stereotaxic coordinates. Amsterdam: Academic.

Peciña S, Berridge KC (2005) Hedonic hot spot in nucleus accumbens shell: where do $\mu$-opioids cause increased hedonic impact of sweetness? J Neurosci 25:11777-11786. CrossRef Medline

Peters J, Kalivas PW, Quirk GJ (2009) Extinction circuits for fear and addiction overlap in prefrontal cortex. Learn Mem 16:279-288. CrossRef Medline

Pisanello F, Sileo L, Oldenburg IA, Pisanello M, Martiradonna L, Assad JA, Sabatini BL, De Vittorio M (2014) Multipoint-emitting optical fibers for spatially addressable in vivo optogenetics. Neuron 82:1245-1254. CrossRef Medline

Quarta D, Borycz J, Solinas M, Patkar K, Hockemeyer J, Ciruela F, Lluis C, Franco R, Woods AS, Goldberg SR, Ferré S (2004) Adenosine receptormediated modulation of dopamine release in the nucleus accumbens depends on glutamate neurotransmission and $N$-methyl-D-aspartate receptor stimulation. J Neurochem 91:873-880. CrossRef Medline

Quiroz C, Luján R, Uchigashima M, Simoes AP, Lerner TN, Borycz J, Kachroo A, Canas PM, Orru M, Schwarzschild MA, Rosin DL, Kreitzer AC, Cunha RA, Watanabe M, Ferré S (2009) Key modulatory role of presynaptic adenosine $\mathrm{A} 2 \mathrm{~A}$ receptors in cortical neurotransmission to the striatal direct pathway. ScientificWorldJournal 9:1321-1344. CrossRef Medline

Richard JM, Berridge KC (2013) Prefrontal cortex modulates desire and dread generated by nucleus accumbens glutamate disruption. Biol Psychiatry 73:360-370. CrossRef Medline

Richard JM, Castro DC, Difeliceantonio AG, Robinson MJ, Berridge KC (2013) Mapping brain circuits of reward and motivation: in the footsteps of Ann Kelley. Neurosci Biobehav Rev 37:1919-1931. CrossRef Medline

Schultz W (2002) Getting formal with dopamine and reward. Neuron 36: 241-263. CrossRef Medline

Strafella AP, Paus T, Fraraccio M, Dagher A (2003) Striatal dopamine release induced by repetitive transcranial magnetic stimulation of the human motor cortex. Brain 126:2609-2615. CrossRef Medline

Stuber GD, Hnasko TS, Britt JP, Edwards RH, Bonci A (2010) Dopaminergic terminals in the nucleus accumbens but not the dorsal striatum corelease glutamate. J Neurosci 30:8229-8233. CrossRef Medline

Taber MT, Das S, Fibiger HC (1995) Cortical regulation of subcortical dopamine release: mediation via the ventral tegmental area. J Neurochem 65:1407-1410. CrossRef Medline

Thompson RH, Swanson LW (2010) Hypothesis-driven structural connectivity analysis supports network over hierarchical model of brain architecture. Proc Natl Acad Sci U S A 107:15235-15239. CrossRef Medline

Vertes RP (2004) Differential projections of the infralimbic and prelimbic cortex in the rat. Synapse 51:32-58. CrossRef Medline

Vidal-Gonzalez I, Vidal-Gonzalez B, Rauch SL, Quirk GJ (2006) Microstimulation reveals opposing influences of prelimbic and infralimbic cortex on the expression of conditioned fear. Learn Mem 13:728-733. CrossRef Medline

Voorn P, Vanderschuren LJ, Groenewegen HJ, Robbins TW, Pennartz CM (2004) Putting a spin on the dorsal-ventral divide of the striatum. Trends Neurosci 27:468-474. CrossRef Medline

You ZB, Tzschentke TM, Brodin E, Wise RA (1998) Electrical stimulation of the prefrontal cortex increases cholecystokinin, glutamate, and dopamine release in the nucleus accumbens: an in vivo microdialysis study in freely moving rats. J Neurosci 18:6492-6500. Medline

Zhang S, Qi J, Li X, Wang HL, Britt JP, Hoffman AF, Bonci A, Lupica CR, Morales M (2015) Dopaminergic and glutamatergic microdomains in a subset of rodent mesoaccumbens axons. Nat Neurosci 18:386-392. CrossRef Medline 